\title{
The effect of the boundary conditions on the simulation of the 4 November 1966 storm over Italy
}

\author{
S. De Zolt ${ }^{1}$, P. Lionello ${ }^{2}$, P. Malguzzi ${ }^{3}$, A. Nuhu ${ }^{1}$, and A. Tomasin ${ }^{4}$ \\ ${ }^{1}$ Department of Physics, University of Padua, via Marzolo 8, 35131 Padua, Italy \\ ${ }^{2}$ Department of Materials Science, University of Lecce, via Arnesano, 70300 Lecce, Italy \\ ${ }^{3}$ ISAC-CNR, via Gobbetti 101, I-40129 Bologna, Italy \\ ${ }^{4}$ Department of Applied Mathematics, University “Ca' Foscari” and ISMAR-CNR, San Polo 1364, 30125 Venice, Italy \\ Received: 31 October 2005 - Revised: 16 December 2005 - Accepted: 20 December 2005 - Published: 8 March 2006
}

\begin{abstract}
This study analyses the extreme event which took place on 4 November 1966, when a storm produced intense and persistent precipitation over northern and central Italy and an extreme surge in the northern Adriatic Sea, causing casualties and huge damages. Numerical simulations with a regional atmospheric model have been performed to reconstruct the phenomenology of the event. Results have been compared with observations. This study shows that the choice of the global fields for initial and boundary conditions is crucial for the quality of the reconstruction. The simulation is reasonably accurate if they are extracted from the NCEP re-analysis, while it is not satisfactory if ERA-40 data are used, though fields have a higher resolution in the ERA40 than in the NCEP set of data. The internal physics of the model plays a smaller role in the reproduction of the dynamics of the event.
\end{abstract}

\section{Introduction}

During the first days of November 1966 Italy was hit by a meteorological event of extraordinary intensity, which caused more than 50 victims and huge damages in terms of economic and artistic goods. A cyclone produced intense and persistent precipitation over northern and central Italy, where exceptional discharge of several rivers and their tributaries caused flooding of towns and countryside. The cases of Florence and Trento are the best known. The sirocco wind produced by the cyclone in the Adriatic basin had an exceptional intensity and duration, determining high waves and the highest surge ever recorded in Venice.

Correspondence to: S. De Zolt

(dezolt@pd.infn.it)

\section{Methodology}

In the present work this event has been studied by performing numerical simulations with the BOLAM (BOlogna Limited Area Model) meteorological model.

The analysis focuses on the SLP (Sea Level Pressure) and precipitation fields. Three different experiments are compared. Two of them, named $E$ and $N$, used different sets of initial and boundary conditions, extracted from ERA-40 and NCEP re-analysis, respectively. This allows to discuss the role of such conditions for the correct description of the event. The third experiment, named $N_{-} c v$, is based on the NCEP re-analysis, and includes the parameterization of the convective instability when the column of air is convectively unstable. This run is meant to test the importance of the model physics in the simulation of this event. Note that both ERA-40 and NCEP re-analysis are based on a similar set of observations and both use a 3-D variational data assimilation method, but at different resolutions. The ERA-40 re-analysis (Gibson et al., 1996) has a T159 spectral resolution, corresponding to about $130 \mathrm{~km}$ resolution, and 60 vertical levels. The NCEP re-analysis (Kalnay et al., 1996) has been performed with a T62 spectral model, corresponding to approximately $210 \mathrm{~km}$ resolution, with 28 levels.

BOLAM is an atmospheric hydrostatic model adopting vertical sigma coordinates and a rotated lat-lon regular grid. It integrates the primitive equations of the dynamics and thermodynamics of the atmosphere using wind velocity, sea level pressure, specific humidity and potential temperature as prognostic variables. The physics of the model includes the description of large scale precipitation and condensation processes, dry convection, vertical diffusion, radiative processes, soil water and energy balance and momentum, humidity and heat surface fluxes. Moreover, it includes a parameterization of the convective instability, recommended to prevent the development of convection on unrealistic spatial scales when the model resolution is coarse. This is the 


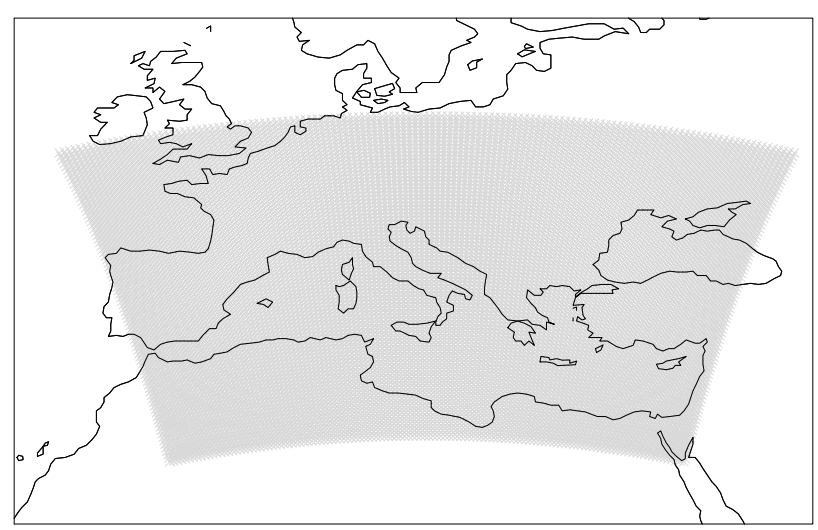

Fig. 1. Computational grid of the BOLAM atmospheric model. The shaded area covers the domain of implementation of the model.

parameterization which has been switched off in the $E$ and $N$ simulations.

The BOLAM model has been implemented over the whole Mediterranean region (Fig. 1). In all experiments the computational grid is the same and has a 0.27 deg spatial resolution in rotated coordinates. Boundary conditions are imposed every $6 \mathrm{~h}$ on the 8 outermost boundaries of the domain, and relaxed toward the centre. Integration starts on 1 November 00:00 UT.

Results of the different simulations have been validated with observations. SLP and precipitation data are available at 34 meteorological stations. More information has been extracted from the hand-made SLP maps of the Italian Air Force Meteorological Service analysis, published for the reconstruction of the event.

\section{Description of the event}

The extreme event is represented by the development and intensification of a baroclinic wave over the European and Mediterranean region. The maps of the $500 \mathrm{hPa}$ geopotential level show a trough, located over the western Mediterranean, which gradually deepens on 3 and 4 November, moves eastward and then vanishes on 5 November. This synoptic situation determined a strong meridional temperature gradient and an intense northward transport of heat and moisture in the middle and lower troposphere. At the surface the sea level pressure (SLP) minimum originated in the western Mediterranean, moved eastward and deepened while crossing the Tyrrhenian Sea. After reaching the Italian peninsula, the cyclone moved north and was absorbed by a deeper pressure minimum crossing northern Europe.

The SLP fields in the observations and in the experiments performed with the regional model show several local minima embedded in a low pressure structure, whose shape is elongated in the north-south direction. The path of two main and long lasting local minima, named A and B, is shown in Fig. 2. Different simulations and observations do not quite

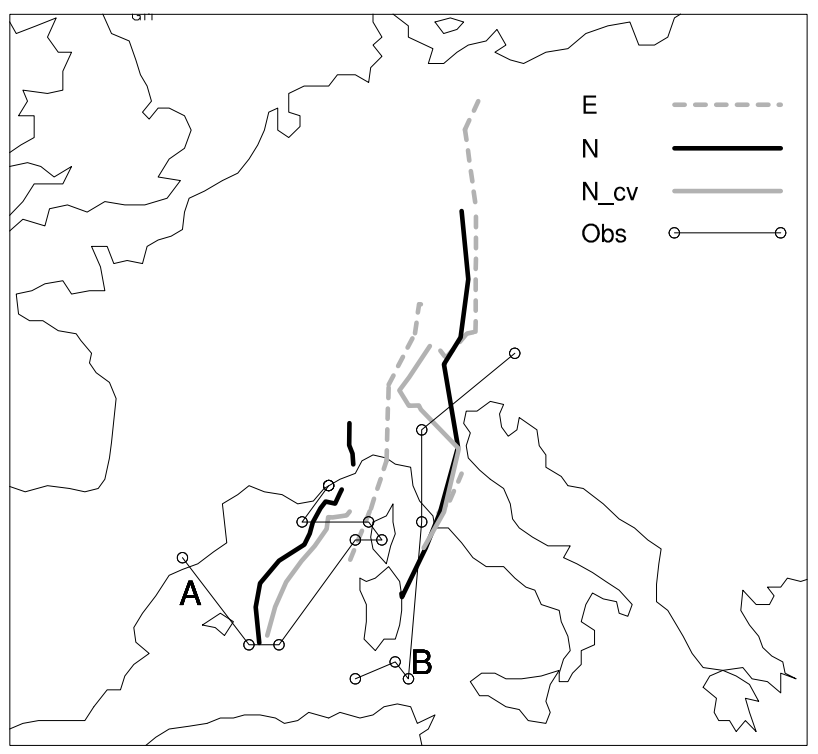

Fig. 2. Trajectories of the low pressure centres named A and B, according to observations and simulations. Some trajectories are broken, when the corresponding minimum is temporarily not detected in the SLP maps.

agree on detailed location and evolution of the local minima. Such complex structure on the regional scale is not present in the ERA-40 and NCEP re-analysis, whose data are used to provide initial and boundary conditions for the different BOLAM simulations, owing to the lower resolution of the global models used. However, from the point of view of a coarse analysis, ignoring subsynoptic details, the evolution of the cyclone is well reproduced by both ERA-40 and NCEP re-analysis. Figure 3 shows the structure of the SLP field on 4 November 00:00 UT, according to the ERA-40 (top panel) and NCEP (bottom panel) re-analysis.

During the evolution of the storm, a high pressure zone was permanently located above the eastern Mediterranean, and, combined with the presence of the cyclone, determined an intense zonal pressure gradient. This feature was responsible for the intense sirocco wind, blowing over the Tyrrhenian Sea during the first part of the storm and on the Adriatic Sea in the later stage. The mountain ridges surrounding this basin further increased the wind speed, determining a surge level of $176 \mathrm{~cm}$ in the Venice city centre, which corresponds to a return time longer than 250 years (Lionello, 2005). Furthermore, sea level height persisted above the $100 \mathrm{~cm}$ level (Canestrelli et al., 2001) for more than 15 hours, flooding a large part of Venice. Data are not available for wave height, which was anyway extraordinary, as waves destroyed coastal defenses in many islands of the Venice lagoon. At the same time the low pressure was not particularly deep and the value reached by the central minimum cannot be considered extreme (Lionello et al., 2002).

A cold front, extending in the meridional direction, was associated with the cyclone, and moved eastward as the storm developed. A warm front, determined by the advection of 

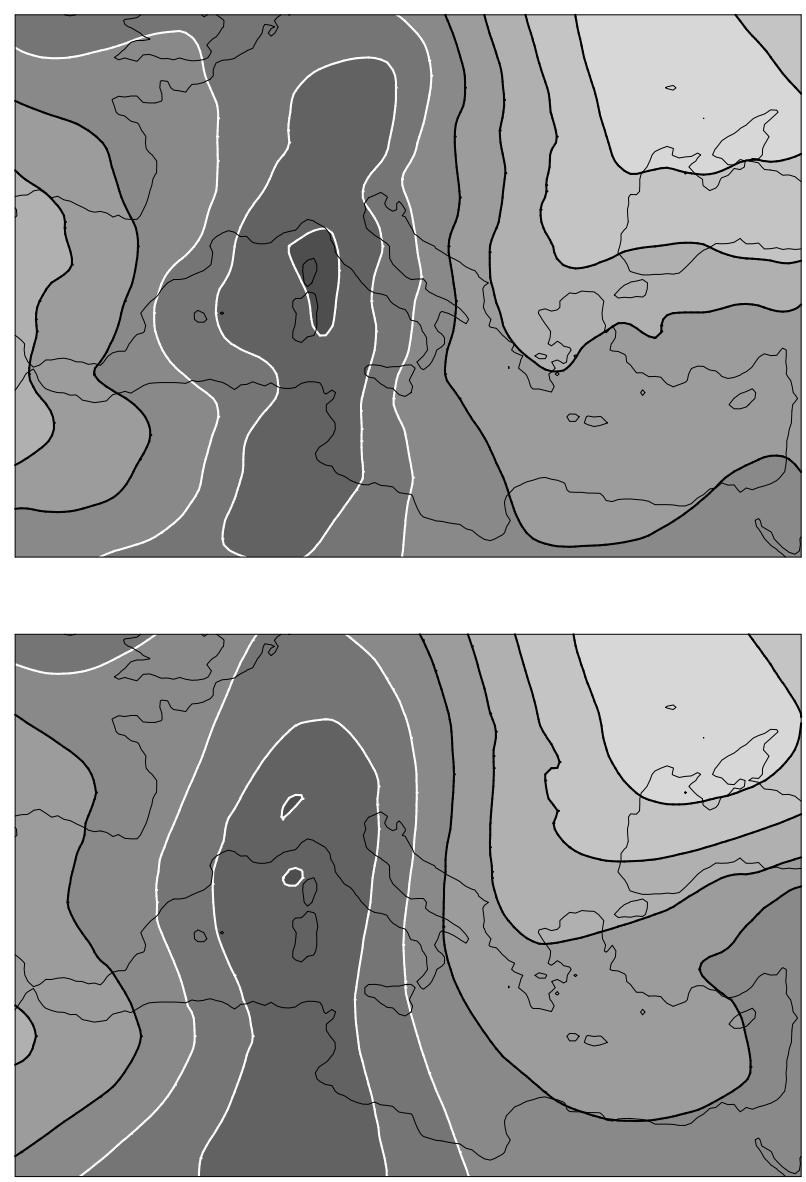

Fig. 3. Mean sea level pressure 4 November 00:00 UT, as described by ERA-40 (top panel) and NCEP (bottom panel) re-analysis. Contour interval is $5 \mathrm{hPa}$; levels lower than $1015 \mathrm{hPa}$ have a white contour; lighter grays correspond to higher values.

warm and humid air by the sirocco wind, moved northwestward along the Adriatic basin. When the cyclone reached the Italian peninsula, the fronts caused intense precipitation all over northern and central Italy, thereby producing severe flooding. Precipitation maxima were located over Tuscany, central Italy and Eastern Alps. In the first two cases, radar maps of the Italian Air Force Meteorological Service (CENFAM, 1967) show that precipitation was associated to the presence of convective cells that formed on the Tyrrhenian sea, along the cold front, and further east, along the coast of central Italy and also inland. Instead, precipitation over the Alps was caused by the orographic uplift of the warm and moist air advected by the wind blowing along the Adriatic basin. In fact, time series of observed wind and temperature in several stations of north-eastern Italy and radiosoundings show that precipitation began after 3 November at 18:00 UT, when winds turned from north-easterlies to southwesterlies and temperatures suddenly raised.

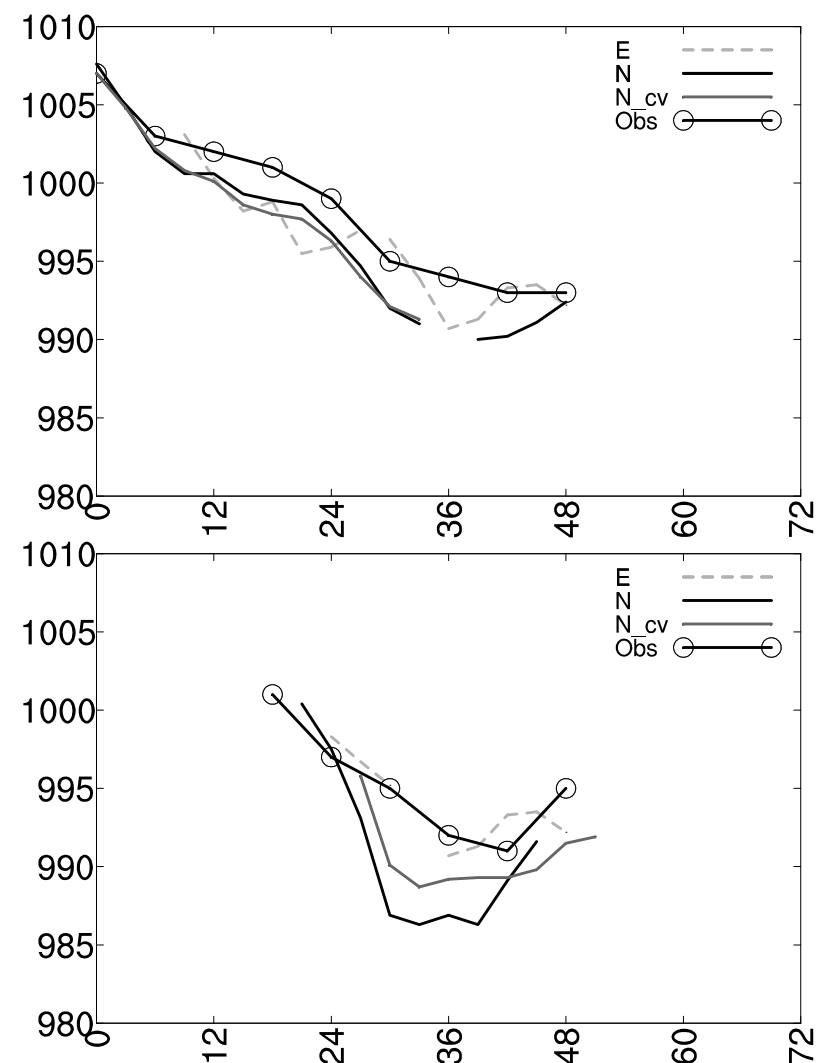

Fig. 4. Time series of the SLP minima in observations and simulations. The panels show separately the minima " $\mathrm{A}$ " (top) and "B" (bottom). The $\mathrm{x}$-axis is time, in hours, from 3 November 00:00 UT to 6 November 00:00 UT. The y-axis is SLP, in hPa. As in Fig. 2, some curves are broken, when the corresponding minimum is temporarily not detected in the SLP maps.

\section{Results}

Differences in the results of the simulations have been analysed and their accuracy has been tested by comparing them with observations.

According to the observations, cyclogenesis takes place in the Gulf of Valencia, with the formation of minimum A (Fig. 2). All simulations miss the first part of the evolution of the minimum A and misplace its formation east of the Balearic Islands. Actually, the simulation $E$ reproduces correctly a weak minimum in the observed location of minimum A, but not its following evolution, as the simulated minimum terminates about one day later, at the French coast, without moving further towards Italy. Therefore, also in simulation $E$ the actual minimum $\mathrm{A}$ is formed in the wrong location. In all simulations A moves too far north and deepens too much, if compared with observations, as it is possible to see in Fig. 4. Nevertheless, as the cyclone path crosses the sea, where little observations are generally available, the quality of the observations might not be accurate in this stage of evolution. In fact, observations describe a quite weak minimum, which is not likely compared with the exceptionality of the intensity of the storm. 


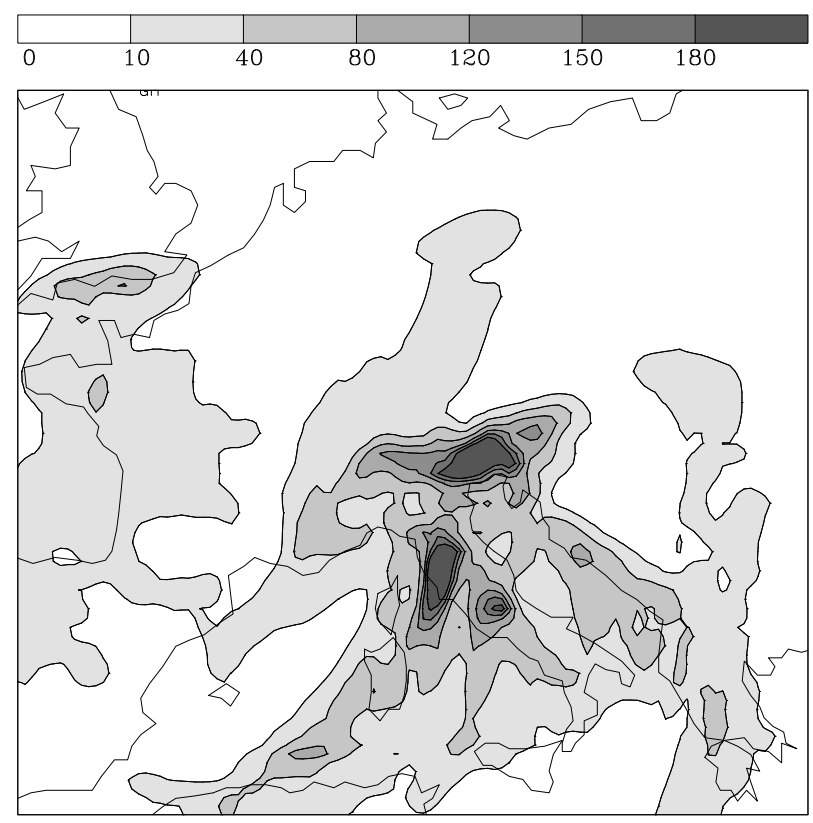

Fig. 5. Distribution of precipitation accumulated between 3 November 00:00 UT and 6 November 00:00 UT, according to the $N$ simulation. Values are in $\mathrm{mm}$.

All simulations reproduce the presence of minimum B. The $N$ and $N_{-} c v$ simulations correctly reproduce its path, crossing the Tyrrhenian Sea and Italy, but miss the first part of its evolution. In these two cases the cyclone deepens too much and moves too fast. In the $E$ simulation the trajectory of B looks unrealistic, as its appearance is incorrectly placed to the north-east, and then it moves over north-eastern Europe in the last stages of its development. These discrepancies are confirmed by the time series of SLP at the single stations (not shown), where the minimum in all simulations is more than $6 \mathrm{~h}$ earlier than the observations.

Major differences between the results of the simulations are determined by the use of the two sets of initial and boundary conditions provided by the ERA-40 and NCEP reanalysis. Significant differences between the two re-analysis in the description of the synoptic situation associated to the event are present both on 1 November 00:00 UT, that corresponds to the initial condition of the BOLAM simulations, and in the following days, as the cyclone reaches its mature stage. This is evident from the structure of the $300 \mathrm{hPa}$ Potential Vorticity (PV) fields, shown in Fig. 7 for the 4 November 00:00 UT. Such differences determine a too fast propagation of the front over the Tyrrhenian Sea and Tuscany in the ERA re-analysis. Nevertheless, because of data assimilation, the shape of the cyclone and the trajectory and depth of its central minimum are similar in both ECMWF and NCEP re-analysis. ECMWF re-analysis has a tendency to develop sharper and deeper features in the SLP field (see Fig. 3), which is consistent with its higher resolution. Note that in both the analysis there is no strong evidence for a new cyclogenesis inside the Mediterranean (which is shown
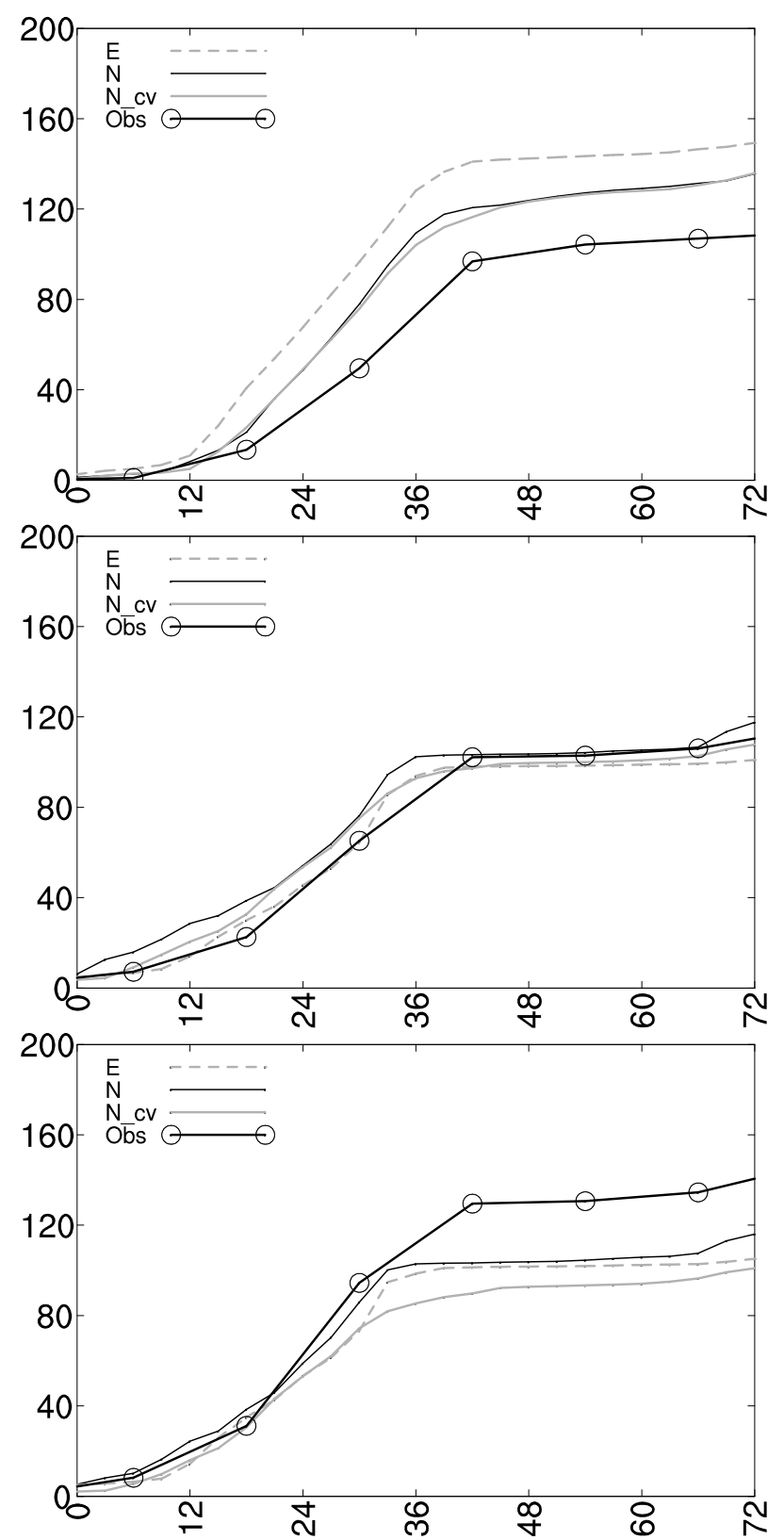

Fig. 6. Time series of accumulated precipitation averaged over stations located in northern Italy (top panel), central Italy (middle panel) and Tuscany. The $x$-axis is time, in hours, from 3 November 00:00 UT to 6 November 00:00 UT. The y-axis is precipitation, in $\mathrm{mm}$.

in the regional model simulation and confirmed by the observations) and the cyclone appears initially above Spain and successively intensifies while passing on the Mediterranean Sea. Also the complicate structure of the SLP, with several minima evolving during the same period, is not present in the global re-analysis, but it is shown by the observations. This points out the importance of using a regional model for the simulation of this event.

All simulations reproduce the three observed precipitation maxima over Tuscany, central Italy and north-eastern Italy 
(Fig. 5), with minor differences in their location. Station observations have been used to evaluate the average accumulated precipitation in these three regions. Precisely, 9 stations are located in north-eastern Italy, 6 stations in Tuscany and 11 stations in Central Italy (which includes also Tuscany). The time series obtained has been compared with the results of the simulations in Fig. 6 . The onset of precipitation, on 3 00:00 UT, and the end of phenomena, on 4 18:00 UT, is captured by all the simulations. Model results overestimate precipitation over northern Italy and underestimate it over Tuscany. If all central Italy is considered the agreement between observations and model results is satisfactory. The simulation $E$ produces the highest values over northern Italy, resulting in this case the less accurate.

Parameterization of convection has not determined an improvement in the simulated precipitation. Note that in central Italy and Tuscany values are higher in the $N$ than in the $N_{c} c v$ case (where precipitation occurs earlier and over sea). The higher precipitation produced by the $N$ simulation in Tuscany and central Italy, where convective processes played an important role for the phenomena, is probably due to the production of convection on unrealistic spatial scales by the model, which has a coarse resolution. In spite of that, in Tuscany, results of the $N$ simulation fit the observations better than the other simulations, even if all of them underestimate precipitation in this region. It would be interesting to analyse results obtained with higher model resolution.

\section{Conclusions}

The reconstruction of the 4 November 1966 storm using the BOLAM model shows the importance of the boundary and initial conditions extracted from global analysis and their effect on the performance of the regional model. This might be completely obvious if the cyclone develops close to the boundary, but in this case its evolution took place in the central part of the domain and boundaries were at least $2000 \mathrm{~km}$ far away, so that spatial scales should allow a strong effect of the physics and dynamics of the model. Moreover initial and boundary conditions were the result of re-analysis projects and were expected to be an accurate reproduction of the real situation both in the NCEP and the ERA-40 sets of data, without major differences between them, but that explained by the different resolution.

In this case, when the NCEP re-analysis is used, the reconstruction of the synoptic evolution and of the precipitation field agrees satisfactorily with observations, even if discrepancies are present. If ERA-40 re-analysis is used, the simulated trajectory of the cyclone is incorrect and larger errors are present in the SLP and precipitation fields. This is caused by a different reconstruction of the synoptic situation associated to the extreme event in the two re-analysis, as it is evident from the structure of $300 \mathrm{hPa} \mathrm{PV}$ field in the two cases on 4 November at 00:00 UT (Fig. 7). In general the ERA-40 reanalysis presents often sharper and more intense features, and in particular, also in the initial condition. This charac-
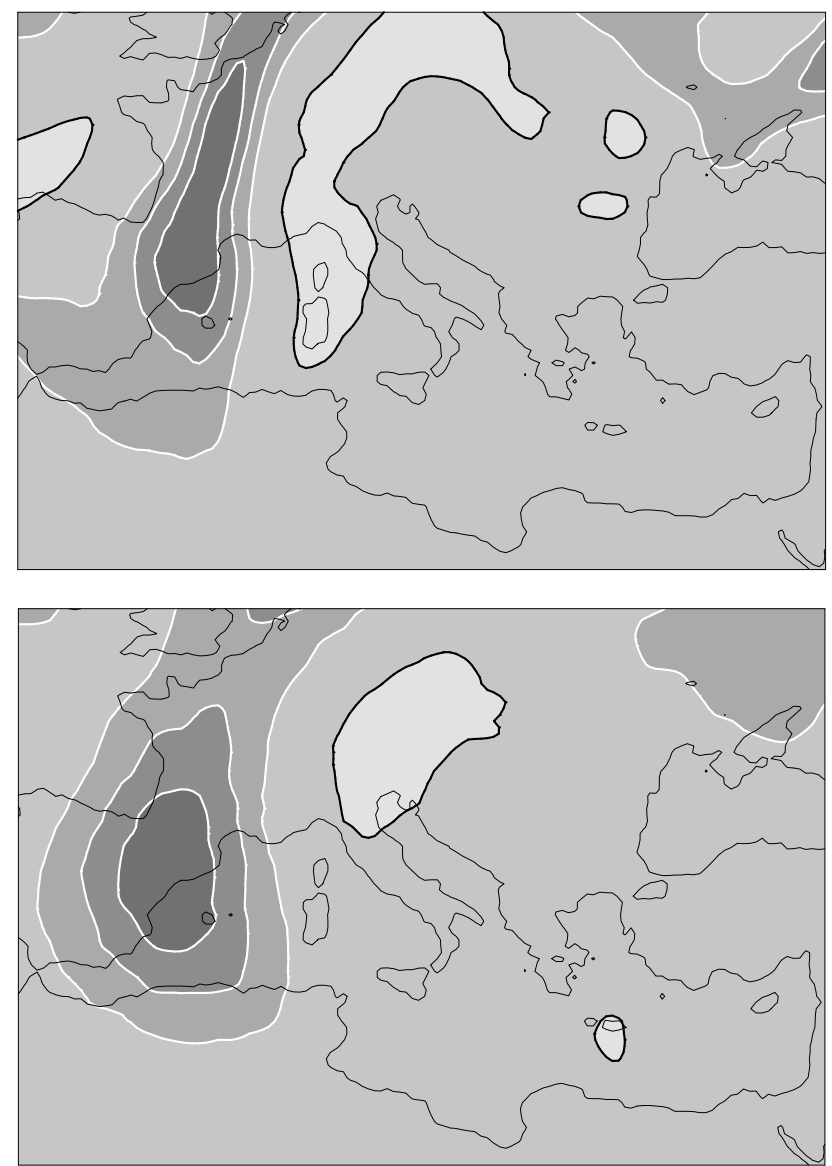

Fig. 7. $300 \mathrm{hPa}$ Potential Vorticity on 4 November 00:00 UT, according to ERA-40 (top panel) and NCEP data (bottom panel). Contour interval is $2 \mathrm{pvu}$; levels higher than 0 pvu have a white contour; lighter grays correspond to lower values.

teristic, more favourable to instability, is likely to have produced the earlier development of the cyclone in simulation $E$ and its consequently lower intensity over central Italy, as the regional model amplifies the initial differences between the two cases. In this experiment the cyclone deepens earlier and follows a more Northern path, and has a smaller impact over central Italy. Owing to the data assimilation the two re-analysis do not show such big differences in the SLP field.

The event was caused by the formation of a cyclone over western Mediterranean. The mesoscale features of this region determined the formation of a complex SLP pattern characterized by the presence of two main local minima above Italy. An intense sirocco wind, characterized by an anomalously long fetch, advected warm and moist air masses towards north-eastern Italy and orographic uplift determined strong precipitation over the Eastern Alps. Phenomena were very intense also over central Italy, where convective cells organized in a rainband extending along the Tyrrhenian Sea.

The role of convection has been studied by comparing results of two experiments in which the associated phenomena are computed with different numerical schemes. In this case, parameterization of convective instability does not 
improve results (different conclusions might be drawn running higher resolution experiments). However, in the case of the 4 November 1966 storm, the role of initial and boundary conditions results more important than this feature of the model for the correct simulation of the event.

Acknowledgements. NCEP re-analysis data are provided by the NOAA CIRES Climate Diagnostic Center, Boulder, Colorado, USA, from their Web site at http://www.cdc.noaa.gov. ERA-40 data are retrieved from the ECMWF MARS archive. The authors are grateful for permission by Aeronautica Militare Italiana to use this link.

Edited by: V. Kotroni and K. Lagouvardos

Reviewed by: anonymous referee

\section{References}

Buzzi, A., Fantini, M., Malguzzi, P., and Nerozzi, F.: Validation of a limited area model in cases of Mediterranean cyclogenesis: surface fields and precipitation score, Meteorol. Atmos. Phys., 53, 137-153, 1994.
Canestrelli, P., Mandich, M., Pirazzoli, P.A. and Tomasin, A.: Wind, depression and seiches: tidal perturbations in Venice (19512000), Città di Venezia, Centro Previsioni e Segnalazioni Maree, Comune di Venezia - CPSM, report 104, 2001.

Consiglio Nazionale delle Ricerche, Centro Nazionale di Fisica dell'Atmosfera e Meteorologia and Ministero della DifesaAreonautica, ITAV, Servizio Meteorologico: Prima documentazione generale della situazione meteorologica relativa alla grande alluvione del novembre 1966, Parte II, Roma, CENFAM, 1967.

Gibson, R., Klberg, P., and Uppala, S.: The ECMWF Re-Analysis (ERA) project, ECMWF Newsl., 73, 7-17, 1996.

Kalnay, E. and Coauthors: The NCEP/NCAR 40-year re-analysis Project, Bull. Amer. Meteor. Soc., 77, 437-471, 1996.

Lionello, P.: Extreme surges in the Gulf of Venice. Present and Future Climate, in: Venice and its lagoon, state of knowledge, edited by: Fletcher, C. and Spencer, T., Cambridge, UK, September 2003, Cambridge University Press, Cambridge UK, 59-65, 2005.

Lionello, P., Dalan, F., and Elvini, E.: Cyclones in the Mediterranean Region: The present and the doubled $\mathrm{CO}_{2}$ climate scenarios, Clim. Res., 22, 147-159, 2002. 